\title{
Determinants of Trustworthiness to Conduct Medical Research: Findings from Focus Groups Conducted with Racially and Ethnically Diverse Adults
}

\author{
Derek M. Griffith, $P h D^{1,2}{ }^{1}$, Emily Cornish Jaeger, $M P H^{7}$, Erin M. Bergner, $M P H, M A^{7}$, \\ Sarah Stallings, $\mathrm{PhD}^{3}$, and Consuelo H. Wilkins, $\mathrm{MD}, \mathrm{MSCl}^{3,4}$
}

'Center for Research on Men's Health, Vanderbilt University 2301 Vanderbilt Place PMB\# 401814, Nashville, TN, USA; ${ }^{2}$ Center for Medicine, Health and Society, Vanderbilt University 2301 Vanderbilt Place PMB\# 401814, Nashville, TN, USA; ${ }^{3}$ Meharry-Vanderbilt Alliance Nashville, USA; ${ }^{4}$ Office of the Vice President for Health Equity, Vanderbilt University Medical Center Nashville, USA.

\begin{abstract}
BACKGROUND: Potential research participants, particularly those from racial and ethnic groups underrepresented in medical research, often decide to participate based on how they judge people, places, and study protocols as trustworthy. And yet, few studies have explored notions of trustworthiness or determinants of trustworthiness from the perspective of potential medical research participants. OBJECTIVE: This paper describes how racially and ethnically diverse potential medical research participants conceptualize what makes researchers, research settings, and research protocols seem trustworthy.

DESIGN: Using a criterion sampling strategy, we recruited African American, Latinx, and White adults for participation in focus groups conducted at a community center servings the Latinx community and at a health clinic that primarily serves the African American community.
\end{abstract}

PARTICIPANTS: A total of 57 African American, Latinx, and White adults

APPROACH: We conducted seven focus groups that explored perceptions and determinants of research, trust, privacy, confidentiality, and research participation. We used a phenomenological thematic analytic approach to explore the determinants of trustworthiness to conduct medical research.

RESULTS: In our effort to identify the factors that affect potential research participants' perspectives on the trustworthiness of medical research, we found three themes: Who is trustworthy to conduct medical research? What influences perceptions of trustworthiness in medical research? And what institutions or settings are trustworthy to conduct medical research?

CONCLUSIONS: These findings highlight that one's willingness to participate in research is driven in part by their perception of the trustworthiness of researchers, research institutions, and the information they are given about potential research opportunities. There are important and modifiable determinants of trustworthiness that may facilitate minority participation in research. We found that research, researchers, and research institutions each have things that can be done to increase trustworthiness and minority participation in research.

Received December 13, 2019

Accepted April 22, 2020

Published online June 3, 2020
KEY WORDS: qualitative research; trust; minority research; focus groups; research participation.

J Gen Intern Med 35(10):2969-75

DOI: $10.1007 / \mathrm{s} 11606-020-05868-1$

(C) Society of General Internal Medicine 2020

$\mathrm{L}$ ack of trust is often framed as something to change among potential research participants rather than highlighting the need for researchers and research institutions to demonstrate that they are trustworthy ${ }^{1,2}$. Trustworthy is the notion that the research, researchers, or research environments are deserving of trust. When research, researchers, or the research environment is worthy or deserving of trust, participants, or potential research participants, are more likely to feel confident that the study is designed to achieve the greatest benefit for society and that the benefits of conducting the research outweigh the potential harms for participants. Consistent with the Belmont Report and other principles of ethical research, trustworthy research does not expose participants to unnecessary risks and includes a clear process to make sure that participants are making informed and voluntary decisions to be part of the study and that the burdens and benefits of the research are distributed fairly across the population ${ }^{3}$. Unfortunately, unethical medical research has been concentrated in marginalized populations such as African American and Latinx populations, and potential research participants who come from these populations may not operate from the assumption that research, researchers, or research environments are trustworthy ${ }^{1,4}$.

More than 50 years ago, Grier and Cobbs ${ }^{5}$ argued that African Americans' suspicions and fears that many sectors of American society are not trustworthy were logical and accurate interpretations of their perceptions and experiences ${ }^{6}$. African Americans, Latinx, and other racial or ethnic minority groups have a long history of receiving inferior quality of medical care even when they have the same level and type of insurance and access to care ${ }^{7-10}$. Scholars also have 
documented numerous incidents of unethical medical research on African Americans, Hispanic-Latinos, and other groups ${ }^{4}$, 11, 12. The United States Public Health Service Study of Untreated Syphilis in the Negro Male is often mentioned as proof that concerns about unethical research are justified, but a number of examples of unethical medical research have occurred well after this study ended and even after the historic Belmont Report set standards for human research protections in $1979^{3}$. Given numerous and well-documented instances of unethical research conducted on racial and ethnic minority populations and other historically marginalized groups $s^{4,11-}$

${ }^{13}$, it is important to reframe notions of trust to recognize that it is in fact logical and rational for members of these populations to approach medical research with some level of skepticism. In addition, increasing numbers of financial relationships between university scientists and industry and other events that cast doubt on the objectivity of individual scientists, their institutions, and the larger system of academic research have fueled skepticism about research trustworthiness ${ }^{14}$.

While there have been considerable efforts to understand and measure trust and mistrust ${ }^{15-17}$, the research exploring how and why potential research participants question the transparency, motives, completeness, goals, and potential harms of research has been limited. Better understanding the factors that influence participants' perceptions of whom they should trust to conduct ethical research would enhance our ability to overcome the complex ways that lack of trust impedes medical research participation. The primary research question guiding this study is what determines trustworthiness to conduct medical research?

\section{METHODS}

This paper presents analyses of data from a larger study designed to explore how African Americans and Latinxs - two groups that have been subject to unethical medical research and who are historically underrepresented in medical research - understand trust in relation to medical research and how that affects their willingness to participate in medical research. The study included focus groups to gather participants' perspectives and forums to educate the lay community about medical research. This study was conducted in collaboration with a community center that specializes in serving the health and social needs of the local Latinx community and with a comprehensive community health center that specializes in serving the local African American community. Neither organization serves exclusively their population of expertise, so people of all racial and ethnic backgrounds are welcome to utilize their services.

\section{Recruitment Strategy and Participants}

This study was conducted in Nashville, TN. Nashville is the state capital and most populous city in the state of Tennessee with approximately 690,000 people. In Nashville, $55.6 \%$ of residents are non-Hispanic White, 27.8\% are Black or African American, $10.4 \%$ are Hispanic or Latino, and $6.2 \%$ identify as being of a different race or ethnicity ${ }^{18}$. Study participants were recruited using a criterion sampling strategy from community sites in metropolitan Nashville via on-site flyers, word of mouth, email listservs, and newsletters. Individuals were eligible to participate if they were (1) 18 years of age or older, (2) able to give informed consent, (3) able to complete a demographic questionnaire, (4) fluent in English or Spanish, and (5) not institutionalized. While the goal was to explore the perspectives of African Americans and Latinxs, if participants who were not members of either of these groups expressed interest, we also allowed them to participate in the groups.

The mean age of the 57 participants was 44 years old, and ages ranged from 18 to 76 years old. Three-fourths of the participants were women, and there were no Latino/Hispanic male participants. Sixty-eight percent of study participants had no prior research involvement (Table 1).

\section{Study Design, Setting, and Data Collection}

The study protocol was approved by the university's Institutional Review Board. Prior to the start of the focus group discussion, we obtained written informed consent and participants were asked to complete a demographic survey. Study staff facilitated the focus groups in community-based sites that were convenient for the participants. Table 2 includes the focus group questions. A total of seven focus groups were completed in order to capture unique and common determinants of trust in research. Each focus group lasted approximately $90 \mathrm{~min}$ and was audio-recorded. Each participant was compensated $\$ 25$ for focus group participation.

Table 1 Demographic Characteristics of Study Participants

\begin{tabular}{ll}
\hline \hline Demographic characteristics & $\boldsymbol{n ( \% )}$ \\
\hline Participants & $57(100 \%)$ \\
Gender & $14(24.6 \%)$ \\
Male & $43(75.4 \%)$ \\
Female & \\
Race/ethnicity & $24(42.1 \%)$ \\
African American & $11(19.3 \%)$ \\
White & $21(36.8 \%)$ \\
Hispanic/Latino & $1(1.8 \%)$ \\
Other & \\
Age range & $17(29.8 \%)$ \\
18-35 & $24(42.1 \%)$ \\
36-55 & $14(24.6 \%)$ \\
56-75 & $2(3.5 \%)$ \\
>76 & \\
Educational attainment & $2(3.5 \%)$ \\
8th Grade or less & $2(3.5 \%)$ \\
Some high school, but did not graduate & $18(31.6 \%)$ \\
High school diploma/GED & $12(21.0 \%)$ \\
Some college or 2-yr degree & $14(24.6 \%)$ \\
College graduate & $9(15.8 \%)$ \\
$>$ college degree & \\
Past research participation & $39(68.4 \%)$ \\
No- have not participated in research &
\end{tabular}

For education, one participant reported both 'Some college or 2-year degree' and 'College graduate' and one participant preferred not to answer 
Table 2 Focus Group Questions

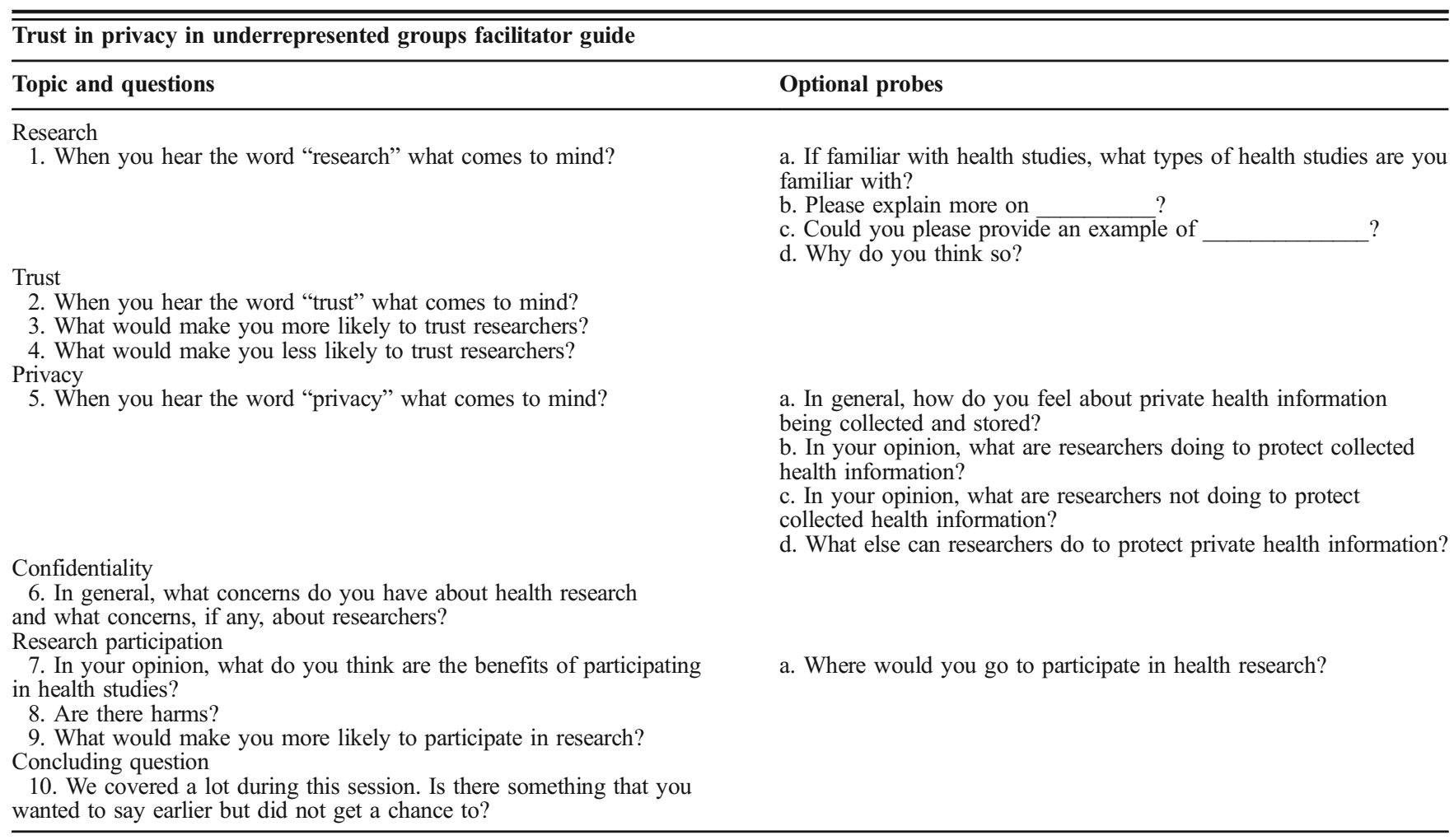

Focus groups were conducted in the participant's preferred language of English or Spanish. Five focus groups were conducted in English. Of the five groups conducted in English, two focus groups were conducted with African Americans, and three focus groups were comprised of racially and ethnically diverse participants. In the three racially and ethnically mixed groups, participants were predominantly White in two groups and most participants were African American in the third group. Two focus groups were conducted with $100 \%$ Latinx participants. These focus groups were conducted in Spanish by a Latina who was a native Spanish speaker and trained focus group moderator.

\section{Data Analysis}

All focus groups were audio-recorded, transcribed verbatim, and when appropriate, translated into English for analysis. Transcribed focus groups were imported into the qualitative data software package NVivo11. Two researchers reviewed the transcripts to check for accuracy, familiarized themselves with the transcripts, and developed an initial codebook from an existing model of conceptions of trust ${ }^{19}$ that informed the larger study and was used to guide the development of the focus group questions and codebook. After developing the codebook, two coders analyzed the same transcript independently to identify additional codes and then convened to develop, refine, and reach consensus on code definitions and rules for applying them. A second focus group was then independently coded by both analysts using the refined codebook and an inter-rater reliability of $78.8 \%$ was achieved. The five remaining focus groups were then divided up among the researchers and coded independently.

A phenomenological, thematic approach was used for anal$\mathrm{ysis}^{20}$. This approach is appropriate when the goal is to use the words of participants to identify, analyze, and report findings that emerge from the data ${ }^{21}$. The data analysis process included five steps: (1) organizing focus group responses into quotes that could be accurately understood outside of the context of the interview; (2) double-checking the quotes and notes; (3) assigning codes to quotes; (4) combining coded restatements across focus groups and participants to form one consolidated document; and (5) analyzing the consolidated document to identify similarities and differences across codes that clustered to classify emerging themes. This analytic approach has been used previously by members of this research team ${ }^{22-25}$.

\section{RESULTS}

In our effort to identify the factors that affect potential research participants' perspectives on the trustworthiness of medical research, we identified three themes (see Table 3):

a. Who is trustworthy to conduct medical research?

b. What influences perceptions of trustworthiness in medical research?

c. What institutions or settings are trustworthy to conduct medical research? 
Table 3 Summary of Results by Theme and Subtheme

\begin{tabular}{|c|c|}
\hline Theme & Subtheme* \\
\hline $\begin{array}{l}\text { Who is trustworthy to conduct } \\
\text { medical research? }\end{array}$ & $\begin{array}{l}\text { - Scientific expertise of the } \\
\text { researcher } \\
\text { - Researcher passion and } \\
\text { enthusiasm for the population of } \\
\text { interest and for the research } \\
\text { - Participants agreeing to } \\
\text { participate in research is an } \\
\text { indication of trust } \\
\text { - White researchers conducting } \\
\text { research in African American or } \\
\text { Latino communities }\end{array}$ \\
\hline $\begin{array}{l}\text { What influences perceptions of } \\
\text { trustworthiness in medical } \\
\text { research? }\end{array}$ & $\begin{array}{l}\text { - Experiences with medical care } \\
\text { can influence medical research } \\
\text { participation } \\
\text { - Informed consent processes } \\
\text { - History of unethical research on } \\
\text { African Americans in particular }\end{array}$ \\
\hline $\begin{array}{l}\text { What institutions or settings are } \\
\text { trustworthy to conduct medical } \\
\text { research? }\end{array}$ & $\begin{array}{l}\text { - Institutions with a stronger } \\
\text { reputation and relationship with } \\
\text { the community } \\
\text { - Prestige or credibility of the } \\
\text { institution where the research is } \\
\text { being conducted } \\
\text { - Institutions being visible and } \\
\text { active in the community }\end{array}$ \\
\hline
\end{tabular}

*Representative quotes for each subtheme appear in the "RESULTS" section

In the remainder of this section, we briefly describe these themes in more detail and use select representative quotes and subthemes to highlight the key points within each theme.

\section{Who Is Trustworthy to Conduct Medical Research?}

Participants noted that trust is influenced not only by the scientific expertise of the researchers, but also by the researchers' knowledge of the history, context, and experience of the study populations. For example, one participant said, "How knowledgeable people around the project are about the people that they're serving and what they're doing. If they present it as though [they have] - I guess the cultural competency, so to speak." In addition, participants noted that the enthusiasm, commitment, or passion of the researcher to help the population of interest and to study the topic of interest influenced how trustworthy the researcher appeared to be. As one participant described, "Passion.... when I talk to you about it [research],... you should make me feel like it's something I should wanna be involved in."

In addition to knowledge about the population and passion for the work, participants noted that just because a researcher has a degree does not mean that the researcher is worthy of trust. The researcher must develop rapport and demonstrate a depth of understanding of the topic of interest and expertise in relevant areas of research to gain the trust of potential research participants. A focus group participant explained, I want "A researcher or an institution that I believe in." Another focus group participant elaborated further on this point and said, "If someone's coming to me with a research [study], you gotta show me some depth. You gotta show me that you've done your own research before you bring it to people. You can't just say, 'Well, I have a $\mathrm{PhD}$ in blah, blah, blah.' That doesn't mean anything. You could have cheated in your classes. "Another participant talked about his perception of clinical trials and the perceived lack of caring from the researcher about the larger cause indicating, "A clinical trial, I don't think is safe... Because they're researching, trying to get something, numbers. I feel like they're trying to get number[s] instead of actually getting a cause taken care of."

Some participants noted that it is important to recognize that simply agreeing to participate in research is an indication of trust in the researcher. For example, one participant said, "I think that is a big indicator of where that trust is because if you don't have to, but you still choose to, then that would indicate a high level of trust between you and whoever you're doing those things for or whoever is presenting you with an opportunity to be a part of the research or a study or something like that." These issues appeared to be relevant regardless of the race or ethnicity of the researcher; however, there were particular ways that race played an important role in shaping whom some participants viewed as trustworthy.

The issue of the race of the researcher only came up in the groups that were comprised exclusively of African Americans. Some African American focus group participants expressed skepticism of White researchers. As one participant described, "I don't have a lot of trust in these folks [White people] just across the board. I generally don't so that transfers into research..." African American focus group participants also were particularly skeptical of the motives of White researchers who were recruiting participants of other racial or ethnic groups. For example, one participant noted, "So if they're doing research [White researchers], that will make me not trust the research. If it was a room full of white people doing the research, then I'm not rocking with it...period."

\section{What Influences Perceptions of Trustworthiness in Medical Research?}

While medical care is separate from medical research, participants sometimes discussed how experiences with care affected their trust in medical research. For example, this exchange illustrates some of participants' concerns with medical research that resulted from their health care experiences:

Facilitator: Okay. Tell me from that experience how your trust in researchers is affected by that experience. Participant: I'm neutral about my trust in research and I'm very neutral in what doctors know and what they actually tell patients.

Facilitator: Okay. So the situation with the type of medicine that they were giving him, did that make you more or less likely to trust researchers? 
Participant: It made me most likely to not trust. Because it did not improve his condition.

Because medical providers often are the ones who also are conducting research and medical research may take place in the same settings as people receive care, it is important to realize how negative experiences with care can adversely affect trust in research and researchers.

In addition to discussing the relationship between medical care and medical research, participants also discussed aspects of the research process that influenced whether they viewed a research study as trustworthy. For example, one focus group participant said, "The consent forms they send are like nine pages long with anything that can go wrong, so I just really don't know what else would make me trust them more." Some felt that the length and legal language included in the consent forms raised concerns about the study focus and goals. Others were skeptical that the research might be taking place for the wrong reasons, such as for profit. Participants expressed concerns that researchers or research institutions receiving monetary incentive to conduct specific types of research may influence the scientific rigor and results. As one focus group participant explained, "Corporations and different things who can have other interests aside from just finding cures and finding ways to improve health. They have interest in what money's getting made, what they already have developed that they're selling... it kind of hurts the process a little bit when on the researching level, whatever they do once it gets to a certain other level, the conversation changes and it isn't always just in the same best interest of public health." The process and perceived motives that were thought to possibly influence the research adversely affected the amount of faith or confidence participants had in medical research.

Furthermore yet unsurprisingly, participants indicated that infamous unethical research studies influenced their level of trust, particularly African Americans. As one participant in the focus groups comprised of African Americans explained, "We, as African Americans were always the pilot test... I know because people have experienced it within my line, my family, my bloodline in [city name]. Yeah." He continued by saying, "So I just think that we've been used and misused a lot of times within the African American community and in the lower parts of the devastation where the devastation lies." Another individual reflected, "I'm thinking about horror stories like the Tuskegee experiment and things like that. Like stuff that for me, were mentioned back in school and were mentioned by my family members..." These participants' perspectives highlight how the history of unethical research that are often discussed as abstract events from a bygone era are actually quite present and relevant to the lives and experiences of many African Americans, particularly in the Southeastern United States. The concerns that arise from these unethical studies demonstrate why medical research would not be blindly trusted, since the research may have directly involved family members, loved ones, or certainly acquaintances of people who are now being asked to participate in medical research.

\section{What Institutions or Settings Are Trustworthy to Conduct Medical Research?}

Lastly, participants talked about how the settings and institutions where research was being conducted affected their interest in participating in research. Participants described how an institution's credentials, reputation, and relationships within the community influence trust in research. Some considered centers such as those affiliated with medical schools or academic medical centers as trustworthy. Others expressed skepticism if research institutions did not have a strong, positive history of being trustworthy. As one African American participant explained, "...the credibility when it comes from [names of academic institutions], those all trustworthy universities. But if it's like a side clinic or a side company or something,... then you won't really trust what's going on because you don't have anything to go off of." Similarly, another focus group participant elaborated, "It has to be a trusted center, a prestigious clinic, or a center that has been doing specific research. We're not going to go to the neighbor [hood clinic] to see how it is."

Participants, particularly those in the majority White focus groups, explained that the credentials and history of research of an institution enhanced the credibility of research conducted at that institution. A participant said, "I trust the credentials of the institution. I don't know about anybody else, but I asked my physician a million questions before I even let you draw blood. It's kind of like credentialing is number one important, but also your history of research." Another participant elaborated, "I think that if it's backed up by some - for example, right now, that we're with the University, a serious university like that, that is prestigious, and not just any - I think that's important."

In addition to the credibility of the institution and the institution's experience with conducting research, if a research institution is visible and active in the community, it is viewed as more trustworthy. One participant explained, "Like he was saying, like building relationship with the community. Because [academic medical center] is everywhere. There's no way that you can miss [academic medical center]. So hence, why so many people trust [academic medical center]...Building those relationships and really being in the community for more than just research." However, what negatively affected how much focus group participants trusted a medical research center was influenced by media reporting of bad medical care outcomes from that institution. One participant explained, "When I was in college, that's where they were sending us to. When I heard about a patient [inaudible] dying, I stopped going." Again, while this was not about research, these clinical outcomes had implications for future clinical and research participation. 


\section{DISCUSSION}

In this paper, we explored the factors that influenced what African American, Latinx, and White adults perceived as trustworthy to conduct medical research. We found three main themes: (a) that there were critical attributes of researchers that influenced whether our focus group participants considered them trustworthy; (b) that medical care, medical research processes, and infamous unethical studies influenced the research that participants considered trustworthy; and (c) that an institution with a history and reputation for being a strong research institution that conducts ethical research and is active in the community was more likely to be viewed as trustworthy.

Researchers often are the reason that participants agree to participate in medical research or not. Our focus group participants noted that the knowledge, expertise, passion, and perceived commitment of the researcher to the research and to the population they are studying and ideally serving by learning new information about them were all key factors that influenced whether or not study participants perceived researchers as people they should trust to conduct research on and with them. Participants noted how important it was to acknowledge that agreeing to participate in research is an important indication of trust in the researcher that should not be overlooked. Furthermore, our findings highlight the critical role that intrapersonal characteristics and interpersonal communication play in fostering confidence that research is being conducted with the highest ethical standards and therefore is worthy of trust.

In addition to the characteristics of the researchers, we also found that research processes and experiences with medical care affected whether or not participants may view future research opportunities as worthy of their trust. Because medical care and medical research are often conducted in the same settings and involve the same people, participants discussed the spillover effects that negative medical care experiences had for their willingness to participate in research. Based on these findings, it is critical to recognize that negative personal experiences with health care have important implications for whether or not medical research is perceived to be worthy of trust and whether or not participants should, therefore, participate in medical research. Further, when the consent forms and other processes seem to be particularly long and overly comprehensive, some participants became skeptical of the motives and goals of the research. If there was some suggestion that there could be a financial incentive, participants expressed concern that these factors may adversely affect the research.

While our data were not designed to explore potential racial or ethnic differences in ways that meet qualitative standards of data trustworthiness ${ }^{26,27}$, African American focus group participants appeared to express more and qualitatively different concerns than Latinx or White participants. African American study participants expressed skepticism of White researchers who recruit participants of other racial or ethnic groups. Some African Americans suggested that if the research team was comprised exclusively of White researchers that they were wary of the motives and goals of the research, others were similarly wary yet open to potentially participating in research. This skepticism is situated squarely in a long and dubious history of unethical research conducted on African Americans where African Americans shared a disproportionate burden of the research yet reaped less than their share of the benefits of medical research ${ }^{1,6,8,11,16,28}$. As was noted in the theme that highlighted aspects of the research process and context, African Americans connected the somewhat abstract history of unethical research conducted on African Americans (e.g., the "Tuskegee" Syphilis study) to their families and communities. These were not events that appeared in history books but injustices that may have affected them and their loved ones. These negative experiences and evidence that all research is not worthy of trust shapes whether or not African Americans are willing to participate in future medical research. The institutional history, reputation, and relationship with the community also played a critical role in whether or not a research institution was considered credible and worthy of being trusted to conduct medical research.

\section{Limitations}

This study provides a useful exploration of how potential research participants view what makes research, researchers, and research institutions trustworthy, but neither our sampling strategy nor analytic approach was designed to achieve saturation within racial or ethnic groups. Thus, the subtle differences noted by race and ethnicity should be viewed as starting points for future research rather than enough data to assert patterns by race or ethnicity. In addition, our study was not designed to explore differences by age, gender, or educational attainment, nor did we track speakers to allow analysis of individual characteristics to facilitate analyses of individual characteristics across groups. The study was conducted in one site and findings may not be generalizable to other locations. Lastly, we recognize that due to the focus group format, some participants may not have elected to share viewpoints that may have differed from others within the group. This limitation of this data collection strategy is outweighed by the benefits of accessing diverse and novel perspectives.

\section{Implications}

Researchers and research institutions should acknowledge and embrace that potential research participants may approach research from a place of trust based on positive individual and collective experiences while others may approach research from more of a place of healthy skepticism. The latter group may still be open and willing to participate in research if their skepticism, fears, and concerns are treated as though they are genuine and honest. Researchers and research institutions that ignore or dismiss these concerns - despite their being consistent with a history of unethical research and researchers - will continue to miss opportunities to achieve the larger aims of 
research because they do not take the effort required to acknowledge and address the legitimacy of these perspectives and concerns.

Corresponding Author: Derek M. Griffith, $\mathrm{PhD}$; Center for Research on Men's Health, Vanderbilt University 2301 Vanderbilt Place PMB\# 401814, Nashville, TN 37240-1814, USA (e-mail: Derek. griffith@vanderbilt.edu).

\section{Compliance with Ethical Standards:}

The study protocol was approved by the university's Institutional Review Board.

\section{Conflict of interest:}

The authors declare that they have no conflict of interest.

\section{REFERENCES}

1. Crawley LM. African-American participation in clinical trials: situating trust and trustworthiness. J Natl Med Assoc 2001;93(12 Suppl):14S.

2. Jacobs EA, Rolle I, Ferrans CE, Whitaker EE, Warnecke RB Understanding African Americans' views of the trustworthiness of physicians. J Gen Intern Med 2006;21(6):642.

3. Adashi EY, Walters LB, Menikoff JA. The Belmont Report at 40: Reckoning With Time. Am J Public Health 2018;108(10):1345-1348.

4. Gamble VN. Trust, medical care, and racial and ethnic minorities. In: Pamies DSRJ, ed. Multicultural Medicine and Health Disparities. New York: McGraw-Hill; 2006.

5. Grier WH, Cobbs PM. Black Rage. New York: Basic Books; 1968.

6. Whaley AL. Cultural mistrust: An important psychological construct for diagnosis and treatment of African Americans. Prof Psychol Res Pract 2001;32(6):555.

7. Smedley BD, Stith AY, Nelson AR. Unequal Treatment: Confronting Racial and Ethnic Disparities in Health Care. Washington, D.C.: National Academies Press; 2003.

8. Byrd WM, Clayton LA. An American Health Dilemma. New York: Routledge; 2000.

9. Byrd WM, Clayton LA. Race, Medicine, and Health Care in the United States: A historical survey. J Natl Med Assoc. 2001;93(3 (Suppl)):11S34S.

10. Byrd WM, Clayton LA. Racial and Ethnic Disparities in Healthcare: A background and history. In: Smedley BD, Stith AY, Nelson AR, eds. Unequal Treatment: Confronting Racial and Ethnic Disparities in Health Care. Washington, DC: The National Academies Press; 2003:455-527.

11. Washington HA. Medical Apartheid: The dark history of medical experi mentation on Black Americans from colonial times to the present. New York: Doubleday; 2007
12. Jaiswal J, Halkitis PN. Towards a more inclusive and dynamic understanding of medical mistrust informed by science. Behav Med 2019;45(2):79-85.

13. Ramos SR, Warren R, Shedlin M, Melkus G, Kershaw T, Vorderstrasse A. A framework for using ehealth interventions to overcome medical mistrust among sexual minority men of color living with chronic conditions. Behav Med 2019;45(2):166-176.

14. Johnston J, Banerjee MP, Geller G. Trustworthy Research Institutions: The Challenging Case of Studying the Genetics of Intelligence. Hastings Cent Rep 2015;45(S1):S59-S65.

15. Hall MA, Dugan E, Zheng B, Mishra AK. Trust in physicians and medical institutions: what is it, can it be measured, and does it matter? Milbank Quarter 2001;79(4):613-639.

16. Benkert R, Cuevas A, Thompson HS, Dove-Meadows E, Knuckles D. Ubiquitous Yet Unclear: A Systematic Review of Medical Mistrust. Behav Med 2019;45(2):86-101.

17. Williamson LD, Bigman CA. A systematic review of medical mistrust measures. Patient Educ Couns 2018;101(10):1786-1794.

18. United States Census Bureau. QuickFacts: Nashville-Davidson (balance), Tennessee; Davidson County, Tennessee. 2018; https://www.census. gov/quickfacts/fact/table/nashvilledavidsonbalancetennessee,davidsoncountytennessee/PST045218. Accessed November 2019.

19. National Academies of Sciences E, Medicine. Applying an Implementation Science Approach to Genomic Medicine: Workshop Summary. National Academies Press; 2016.

20. Fereday J, Muir-Cochrane E. Demonstrating rigor using thematic analysis: A hybrid approach of inductive and deductive coding and theme development. Int J Qual Methods 2008;5(1):80-92.

21. Braun V, Clarke V. Using thematic analysis in psychology. Qual Res Psychol 2006;3(2):77-101.

22. Cornish EK, McKissic S, Dean D, Griffith DM. Lessons learned about motivation from a pilot physical activity intervention for African American men. Health Promot Pract 2017;18(1):102-109.

23. Griffith DM, Cornish EK. "What defines a man?": Perspectives of African American men on the components and consequences of manhood. Psychol Men Masculinity 2018;19(1):78-88

24. Griffith DM, Cornish EK, Bergner EM, Bruce MA, Beech BM. "Health is the ability to manage yourself without help”: How older African American men define health and successful aging. The Journals of Gerontology: Series B. 2017.

25. Griffith DM, Cornish EK, McKissic SA, Dean DAL. Differences in perceptions of the food environment between African American men who did and did not consume recommended levels of fruits and vegetables. Health Education \& Behavior. 2016.

26. Lincoln YS, Guba EG. Naturalistic Inquiry. Beverly Hills, CA: Sage; 1985.

27. Creswell JW. Research Design: Qualitative, Quantitative, and Mixed Methods Approaches. Sage publications; 2013.

28. Gamble VN. Under the shadow of Tuskegee: African Americans and health care. Am J Public Health 1997;87(11):1773-1778.

Publisher's Note: Springer Nature remains neutral with regard to jurisdictional claims in published maps and institutional affiliations. 